\title{
Vochysia awasensis (Vochysiaceae), nueva especie de los Bosques Montanos del Chocó ecuatoriano
}

\author{
Vochysia awasensis (Vochysiaceae), new species from the Mountain Forests \\ from the Ecuatorian Chocó
}

\author{
Isau Huamantupa Chuquimaco
}

Herbario (CUZ), Facultad de Ciencias Biológicas, Universidad Nacional San Antonio Abad del Cusco, Prolongación Av de la Cultura s/n, Cusco, Perú.

Email: andeanwayna@gmail.com $\begin{array}{ll}\text { Presentado: } & 21 / 07 / 2011 \\ \text { Aceptado: } & 23 / 12 / 2011\end{array}$ Publicado online: $08 / 02 / 2012$

\section{Resumen}

Vochysia awasensis I. Huamantupa, es descrita e ilustrada como especie nueva de la familia Vochysiaceae, perteneciente a la sect. Ciliantha Stafleu subsect. Megalanthae Stafleu, proviene de los bosques montanos de la región biogeográfica del Chocó en el noroeste de Ecuador. Presenta mayor afinidad a V. megalantha y $V$. duquei, pero a su vez se distingue de estas por presentar el peciolo sésil poco conspicuo y decurrente, limbo foliar de 17-33,5 (-35) x 4,5-10 cm; pétalos florales entre 2,4-3 x 0,6-0,9 cm.

Palabras clave: Vochysia, Reserva étnica, Awá-Camumbí, Chocó, Ecuador

\section{Abstract}

Vochysia awasensis I. Huamantupa, is described and illustrated as new species of the family Vochysiaceae, belonging to the sect. Stafleu Ciliantha subsect. Megalanthae Stafleu, which comes from the montane forests of the Choco biogeographical region in the northwestern of Ecuador. The new species differs from its relatives $V$. megalantha and V. duquei, by its inconspicuous and recurrent petiole, blade $17-33,5(-35) \times 4,5-10 \mathrm{~cm}$, and petals $2,4-3 \times 0,6$ to $0,9 \mathrm{~cm}$.

Keywords: Vochysia, Reserva étnica, Awá-Camumbí, Chocó, Ecuador

\section{Introducción}

Gran parte del noroeste de Ecuador está comprendido en la región biogeográfica del Chocó, área que se extiende a lo largo de la costa pacífica de Suramérica, desde el sur de Panamá hasta el noroccidente ecuatoriano. Esta región contiene los bosques más húmedos de América y es reconocida como una de las áreas de mayor diversidad biológica en el planeta (Myers et al. 2000), y una de las más amenazadas por el potencial de recursos que representa para los intereses económicos (Plan de salvaguarda étnica del pueblo indígena Awá 2010). En el lado ecuatoriano el territorio Awá comprende aproximadamente 116640 ha. Según Sierra (1999), la reserva étnica del Awá-Camumbi se ubica dentro del bosque siempre verde piemontano, sector de las estribaciones de la cordillera occidental en la subregión norte; caracterizada por presentar alta humedad con precipitaciones anuales de 4000 a $5000 \mathrm{~mm}$ (Guzman 2011). Entre los pocos estudios florísticos destaca el de árboles silvestres manejados en áreas de potreros por Guzmán (2011) quién registra 122 especies en 26 ha, siendo las Lauraceae, Melastomataceae, Moraceae, Urticaceae y Fabaceae las familias más ricas; en cuanto a especies sobresalen el Cedro (Cedrela odorata), Copal (cf. Dacryodes - cf. Protium), Pepa Mono (cf. Hippocrateaceae - cf. Sapotaceae), Chanul (cf. Humiriastrum), Corocillo (cf. Sloanea), Corozo (Pouteria sp.), Caimitillo (Pouteria sp.) y Puegunde (Pseudolemdia cf. laevis). Más para la familia Vochysiaceae se menciona a (cf. Vochysiaceae), con poca importancia.

El género neotropical Vochysia abarca alrededor de 135 especies (Stafleui 1958, Litt 2004, Marcano-Berti 2005), con 12 especies registradas para Ecuador (Jørgensen \& León-Yánez 1999, Neill \& Ulloa 2011). La especie nueva descrita a continuación es la segunda especie de Vochysia a ser descrita sobre la base de colecciones provenientes de Ecuador.

\section{Taxonomía}

\section{Vochysia awasensis I. Huamantupa, sp. nov.}

$$
\text { (Figs. } 1 \text { y 2) }
$$

TIPO: ECUADOR. Provincia, Carchi. Cantón. TulcanChical. Parroquia. Tobar Donoso, reserva étnica Awá-Camumbí. Bosque montano bajo pluvial, suelo pantanoso. 20-29 Julio 1991; "María Teuig", entre $1700-1900 \mathrm{~m}, 78^{\circ} 16^{\prime} \mathrm{S}, 00^{\circ} 53^{\prime} \mathrm{N}$; C. Quelal, C. Aulestia y F. Nastacuáz 224 (Holotipo: MO; Isotipo: QCNE).

Vochysiae duquei affinis, sed stipulis majoribus, foliis in quoque verticillo quatuor, plus $15 \mathrm{~cm}$ longioribus, decurrentibus et inflorescentiis quam $15 \mathrm{~cm}$ majoribus recedit; a V. megalantha stipulis majoribus, foliis subtus glabris (nec ferrugineo-pubescentibus) differt.

Árbol de $8-20 \mathrm{~m}$. Ramas jóvenes glabras a tenuemente ciliado-hispídulas en los ápices, tetrágonas o subtetragonas hasta levemente anguladas, ramas viejas se muestra en subteretes con la corteza persistente banquecino-marrón. Estípula $3-5 \mathrm{~mm}$ de largo, 2,5 - $4 \mathrm{~mm}$ de largo en la base, blanquecino, glabro, triangular-sagitado levemente acuminada. Hojas $4-6$ verticiladas, mayormente 4; peciolo inconspicuo, acanalado, decurrente con el limbo foliar; limbo espatulado, elíptico u obovado, 17 - 33,5 $(-35) \times 4,5-10 \mathrm{~cm}$; ápice redondeado, levemente acuminado a veces retuso; base aguda decurrente con el pecíolo; haz glabro, hasta con cilios escasamente esparcidos; envés glabro; vena media impresa por la haz con cilios cortos ralamente esparcidos, sobresaliente en el envés, cilios rojizos dispuestos ralamente, hasta 3,5 mm de ancho en la base de la hoja; venas laterales 19 - 28 en cada semilimbo, con $0,8-1,9 \mathrm{~cm}$ de separación 


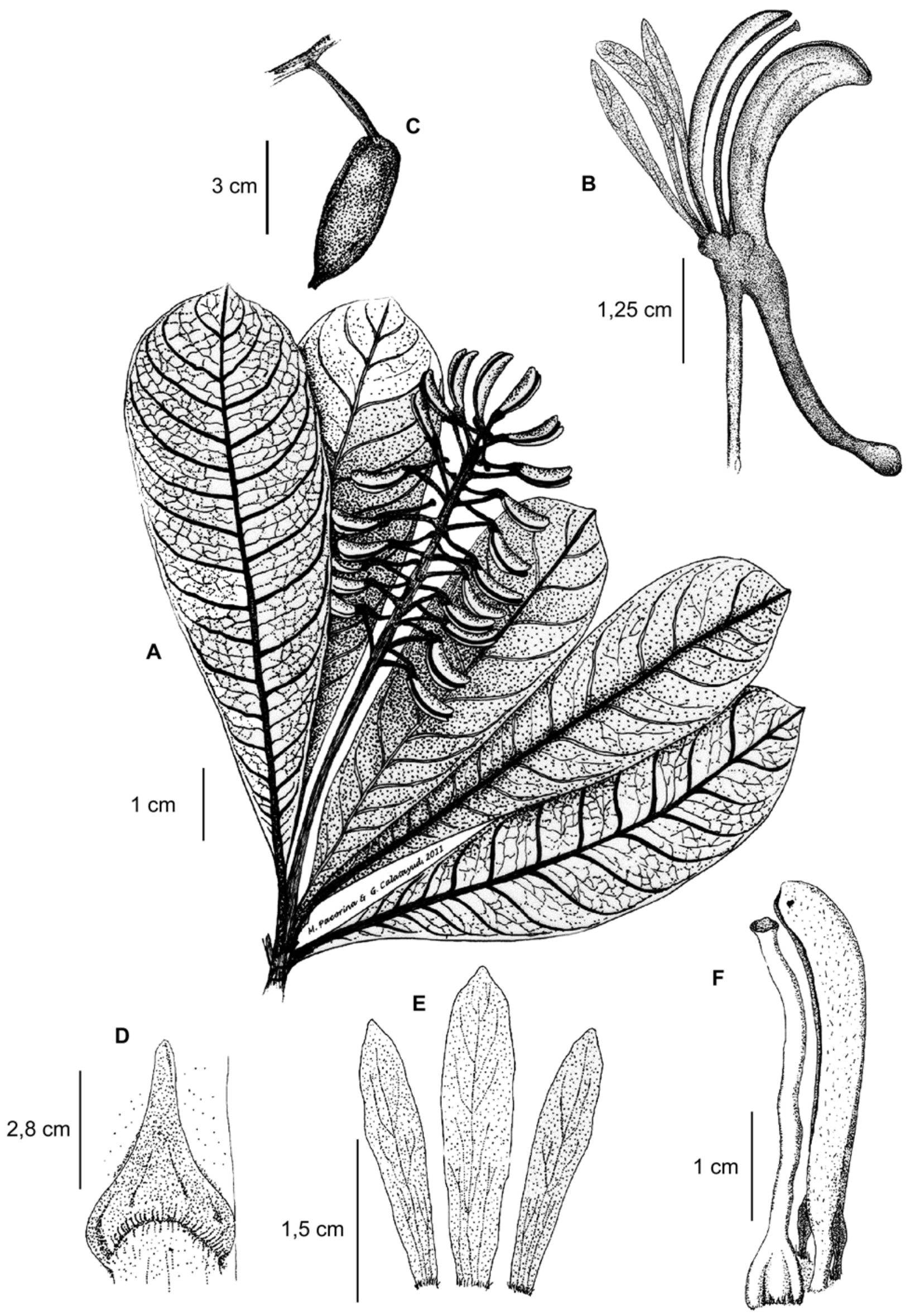

Figura 1. Vochysia awasensis I. Huamantupa. sp nov. A. Rama florifera, B. Flor, C. Fruto, D. Estípula, E. Pétalos y F. Estilo, Estambre y Estaminodios. Ilustración de M. Pacorina y G. Calatayud, basado en el tipo, Quelal et al. 224. 


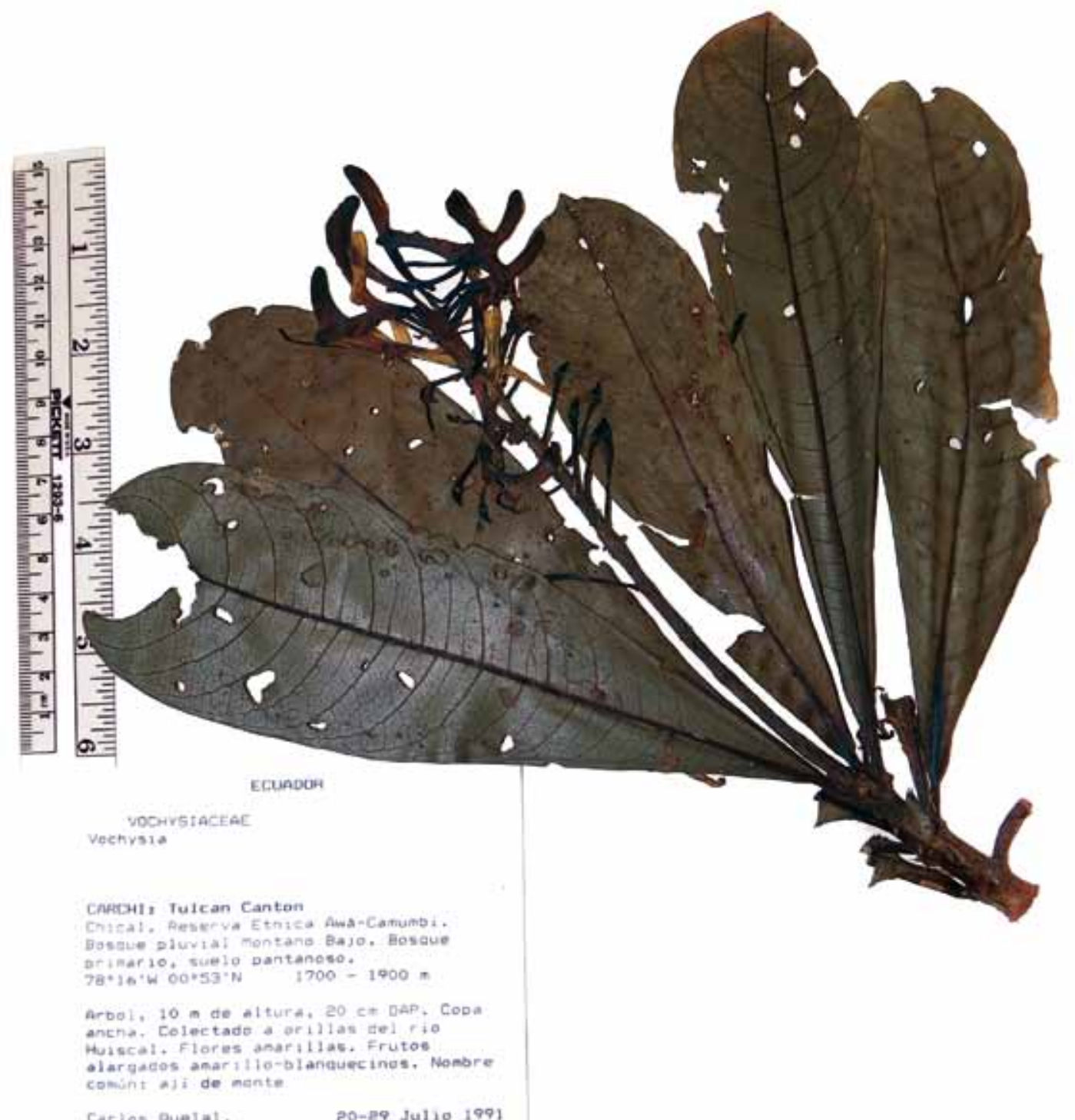

Figura 2. Vochysia awasensis I. Huamantupa sp. nov. Rama florífera. Foto, I. Huamantupa.

entre ambas en la zona media del limbo, 3- $7 \mathrm{~mm}$ en la base y ápice del limbo, forman ángulo de $60-90^{\circ}$ con la vena media, subimpresas por la haz, notorias por el envés, casi inconspicuas en los bordes del envés, cilios cortos ralamente dispuestos, venas laterales secundarias entre las primarias de $1-2$, con frecuencia 1 ; vénulas abundantes; vena marginal poco conspicua en haz y envés, de $1-3 \mathrm{~mm}$ del borde. Inflorescencia terminal solitaria, cilíndrica mayor a $15 \mathrm{~cm}$, poco densa a pauciflora, eje floral ralamente villloso a ciliado-tomentuloso; cincinos con 1 flor, pedúnculo y pedicelo $1,5-4 \mathrm{~cm}$ de longitud, glabrescentes a muy escasamente ciliadas. Yema floral 0,8 - 1,5 cm, subcurvado, ápice acuminado obtuso, ralamente ciliado. Flores amarillas en conjunto con el sépalo espolonado $2,8-5,2 \times 0,6-1,2 \mathrm{~cm}$, curvado abarquillado incluyendo al sépalo dorsal; espolón cilíndrico curvado, ápice redondeado, 1,4-2,2 cm de longitud, forman un ángulo de $40-80^{\circ}$ con el pedicelo, muy ralamente ciliado; sépalo dorsal $2-2,8 \mathrm{~cm}$, escasamente ciliado mayormente en los bordes, sépalos menores $2-2$ x 1,9-4 mm, borde ciliado; pétalos 3, desiguales; oblongo lanceolados, glabros en general, ralamente ciliado-piloso en la cara abaxial de la vénula media y los bordes, pétalo central mayor $2,4-3 \times 0,6-0,9 \mathrm{~cm}$, en la antesis raramente excede al sépalo dorsal, pétalos menores \pm $2,4 \mathrm{~cm}$ de longitud, glabros en general, cilios en la base, son menores en tamaño al sépalo dorsal. Estambre 2,4-2,8 cm de longitud, levemente curvado; filamento corto $3-4 \mathrm{~mm}$ de longitud, ralamente ciliado especialmente en la base; anteras $2-2,4 \mathrm{~cm}$, conduplicadas, levemente incurvadas más anchas en ápice, cada lado de la antera al exponerse $\pm 2,5 \mathrm{~mm}$, glabras en general con cilios esparcidos especialmente en los bordes. Estaminodios 2, lanceolado elípticos 3-4,1 mm, en la base con agrupación de cilios. Ovario triloculado 2 - 3,5 mm, glabro. Estilo 2,3-2,65 cm; estigma terminal capitado. Fruto capsula verruculosa, glabra, oblongo obovada 3,9-7 x 1,2-2,4 cm.

\section{Material adicional analizado}

ECUADOR. Provincia, Carchi. Cantón, Tulcan. Parroquia, Tobar Donoso, Sector Sabalera. Reserva étnica Awá-Camumbí. Bosque muy húmedo pre montano. "María Teuig". 19-28 junio 1992. G. Tipaz, J. Zuleta \& N. Guanga 1330 (MO, QCNE); 
Sector Sabalera. Reserva étnica Awá-Camumbí. Bosque muy húmedo pre montano. Orilla del río botella. "María teuig", 19-28 junio 1992. G. Tipaz, J. Zuleta \& N. Guanga 1353 (MO, QCNE).

Se compara con las especies afines a $V$. duquei Pil. y $V$. megalantha Stafl, diferenciándose de $V$. awasensis por lo siguiente: $V$. duquei, árboles generalmente de gran tamaño, estipulas 0,5 $-1,8 \mathrm{~mm}$. Hojas en verticilos tetrámeros; limbo foliar no excede los $15 \mathrm{~cm}$ de longitud; pecíolo $0,5-1,5 \mathrm{~cm}$; venas primaria y secundarias bastante marcadas en la haz y el envés, en cada semilimbo el número de venas principales no excede 20 nervios. Inflorescencias cortas menos de $15 \mathrm{~cm}$, pénduloides. Flores en conjunto con el espolón $1,5-3 \mathrm{~cm}$; espolón máximo $1,6 \mathrm{~cm}$; pétalos pilosos pubescentes el mayor máximo $2 \mathrm{~cm}$. Ovario piloso ferrugíneo. Fruto hasta $3,8 \mathrm{~cm}$.

$V$. megalantha, ramas con estipulas y peciolos ferruginopubérulos; estipulas hasta $2 \mathrm{~mm}$. Hojas elípticas obovadas; peciolo hasta $1,5 \mathrm{~cm}$; limbo foliar máximo hasta $15 \mathrm{~cm}$; base cuneada; envés ferrugíneo hirsuto, más abundante en las nervaduras primaria y laterales; en conjunto las ramitas al secar se tornan oscuras con las hojas rojizas. Flores, incluido el espolón $2,5-3,5 \mathrm{~cm}$, pedúnculo y pedicelo floral máximo $2,5 \mathrm{~cm}$; filamento hasta $1 \mathrm{~mm}$ de longitud, antera $0,3-0,4 \mathrm{~cm}$.

De acuerdo con el esquema taxonómico de Vochysia de Stafleu (1948) Vochysia awasensis se ubica en Vochysia sect. Ciliantha, subsect. Megalanthae (Stafleu 1948) y la subsect. Megalanthae, se caracteriza por presentar la corteza (epidermis vieja) no exfoliante, hojas en verticilos tetrámeros a más, glabras; inflorescencia terminal pilosa; flores espolonadas de $1,5-3,5 \mathrm{~cm}$ de longitud, espolón nunca más largo que el estandarte (4to sépalo); pétalos 3; filamento muy corto de $0,1-0,3 \mathrm{~cm}$, estaminodios largos y desarrollados.

\section{Ecología y distribución}

Hasta la fecha Vochysia awasensis está registrada solamente para los bosques montanos del Chocó en el noroeste de Ecuador, en la reserva Étnica y Forestal Awá, ubicada entre las provincias de Esmeraldas y Carchi, y colinda con su similar en Colombia que posee el mismo nombre. Por tanto $V$. awasensis, muy posiblemente también se encuentre en el lado del Chocó colombiano, entre 1500 - $2500 \mathrm{~m}$ de altitud. Se observó floración entre los meses de junio a julio y la fructificación entre julio y agosto.

\section{Conservación}

Afortunadamente Vochysia awasensis habita en los bosques del Chocó ecuatoriano, dentro de la reserva forestal y étnica Awá, la cual forma parte de la iniciativa de conservación del corredor Chocó-Manabí, conllevada por Conservación Internacional. Cabe resaltar que aproximadamente el $65 \%$ de los bosques de la reserva étnica Awá son primarios, por lo cual el hábitat de $V$. awasensis se encuentra con buen estado de conservación. Sin embargo estos bosques no son ajenos a amenazas propiciadas por el hombre, siendo las principales la extracción ilegal de madera, la ampliación de zonas agrícolas y conflictos sociales entre los indígenas, los negros y mestizos (Guzman 2011). De acuerdo a los datos conocidos de $V$. awasensis, y los criterios dispuestos por la Lista Roja de la UICN (2001), consideramos que esta especie debe ser listada en la categoría "Casi Amenazada" (NT).

\section{Etimología}

El epíteto está dedicado en honor al grupo étnico Awá, autodenominado "Gente de la montańa o la selva", que ha habitado milenariamente gran parte de la región biogeográfica del Chocó en Ecuador y Colombia.

\section{Agradecimientos}

Al herbario nacional de Ecuador QCNE, que a través de la Blga. Elsa Toapanta nos brindó las facilidades de acceso a las colecciones. A Robin Foster por su apoyo en la obtención de una pasantía en el Field Museum de Chicago y el MO, igualmente a Tyana Watcher y Juliana Phillips, con su apoyo logístico durante mi estadía en USA. A Olga Martha Montiel y al Dr. Ronald Liesner, por su apoyo logístico y en la revisión de las Vochysiaceae en el MO. A Janeth Santiana y Jesús Muñoz por la bibliografía facilitada; Al Dr. Henk Van der Werf, por su apoyo en la diagnosis del latín.

\section{Literatura Citada}

Guzman N. L. 2011. Los árboles aislados en el territorio del pueblo indígena Inkal Awá: Un estudio de caso en la frontera entre Colombia y Ecuador. Tesis para optar el grado de Máster en Conservación y Gestión del Medio Natural. Universidad Internacional de Andalucía UNIA. Huelva, España. pp 57.

Jørgensen P.M. \& S. León-Yánez (eds.) 1999. Catalogue of the vascular plants of Ecuador. Monogr. Syst. Bot. Missouri Bot. Gard. 75: 1-1181.

Litt A. 2004. Vochysiaceae. Pp. 396-398 En: Smith, N., S. A. Mori, A. Henderson, D.W. Stevenson \& S.V. Heald (eds.). Flowering Plants of the Neotropics. New York Botanical Garden, New York.

Marcano-Berti L. 2005. Vochysiaceae. Pp. 500-524 En 191 En Berry, P.E., K. Yatskievych \& B.K. Holst, (eds). Flora of the Venezuelan Guayana. Vol. 9. Missouri Botanical Garden Press, St. Louis.

Myers N., Mittermeier, R.A., Mittermeier, C.G., et al. (2000) Biodiversity hotspots for conservation priorities. Nature 403, $853-858$

Neill D. \& C. Ulloa 2011. Adiciones a la flora del Ecuador: segundo suplemento, 2005-2010. Fundación Jatum Sacha. QuitoEcuador. pp 202.

Plan de salvaguarda étnica del pueblo indígena Awá. 2010. Documento promulgado por el Auto 004 del 26 de enero de 2009. pp 80.

Sierra R. 1999. Propuesta preliminar de un sistema de clasificación de la vegetación para el Ecuador continental. Proyecto INEFAN/GEF-BIRF y EcoCiencia. pp 192.

Stafleu F. A. 1948. A monograph of the Vochysiaceae. I. Salvertia and Vochysia. Recueil des Travaux Botanique Néerlandaise 41: 398-540.

UICN. 2001. Categorías y Criterios de la Lista Roja de la UICN. Versión 3.1. Preparado por la Comisión de Supervivencia de Especies de la UICN. UICN, Gland, Suiza y Cambridge, Reino Unido. 